J. Clin. Chem. Clin. Biochem.

Vol. 17, 1979, pp. 757-765

\title{
Lectine als Reagentien zur Differenzierung von Enzymen im Serum
}

\section{Lectine als Reagentien, I. Mitteilung}

Von K. Lorentz, Barbara Flatter und F. W. Kolle ${ }^{1}$ )

Aus dem Institut für Klinische Chemie (Direktor: Prof. Dr. K. Lorentz) der Medizinischen Hochschule Lübeck

(Eingegangen am 3. Mai/1. August 1979)

Dem Gedenken an Professor Dr. Gábor Szász gewidmet

Zusammenfassung: Lectine aus Canavalia ensiformis, Phaseolus vulgaris und Triticum vulgare reagieren mit Arylamidase, alkalischer Phosphatase, $\gamma$-Glutamyltransferase und Cholinesterase menschlicher Seren unter Bildung enzymatisch aktiver, zumeist unlöslicher Komplexe. Dies geschieht mit Arylamidase, alkalischer Phosphatase und Cholinesterase bei Gesunden intensiver als beim Vorliegen von Leberkrankheiten und bösartigen Tumoren. Arylesterase wird nur von Concanavalin A stärker gebunden. Die Reaktion der genannten Enzyme mit Lectinen aus Ricinus communis, Arachis hypogaea, Helix pomatia, Glycine max, Dolichos biflorus und Ulex europaeus verläuft schwächer und nimmt in dieser Reihenfolge ab. Obwohl multiple Formen mit geringem Gehalt an N-Acetylneuraminsäure von niedrigen Lectinkonzentrationen bevorzugt gebunden werden, verbessert eine Vorbehandlung mit Nęuraminidase nur die Reaktion von Lectin aus Glycine max. Da höhere Konzentrationen auch rasch wandernde Fraktionen mit hohem $\mathrm{N}$-Acetylneuraminsäure-Gehalt erfassen, ist, wie bei den quantitativen Untersuchungen, keine sterische Behinderung der Reaktion von Lectinen mit Sialoenzymen anzunehmen.

\section{Lectins as reagents for the differentiation of serum enzymes Lectins as reagents, $I$.}

Summary: Lectins from Canavalia ensiformis, Phaseolus vulgaris, and Triticum vulgare react with arylamidase, alkaline phosphatase, $\gamma$-glutamyltransferase, and cholinesterase of human sera by formation of enzymatically active, mostly insoluble complexes. Arylamidase, alkaline phosphatase, and cholinesterase react more intensely in sera of healthy people than in sera of patients with liver and neoplastic diseases. Arylesterase is bound to a distinct degree only by concanavalin $\mathbf{A}$. The enżymes mentioned above also react slightly with the following lectins in order of decreasing intensity: Ricinus communis, Arachis hypogaea, Helix pomatia, Glycine max, Dolichos biflorus, and Ulex europaeus.

Though multiple forms containing less sialic acid are favourably bound, preincubation with neuraminidase does not improve the reaction except with soybean lectin. Since higher concentrations of lectins react also with fast moving fractions of high sialic acid content, no steric hindrance of the binding between lectins and sialoenzymes is supposed, as concluded from determination of the total enzyme activity.

\section{Einführung}

Die Bindung von Lectinen durch bestimmte Glykosylreste besitzit grundsätżliche Bedeutung für die Analytik von Glykoproteinen (1-3), doch wurde sie erst vereinzèlt zur Untersuchung mikrosomaler Enzyme in Humanseren eingesetzt (4-6). Die folgende Mitteilung beschreibt daher die Eignung von zehn Lectinen, multiple
Formen von fünf Enzymen im Serum zu differenzieren. Weiterhin berichtet sie über den diagnostischen Wert dieses Verfahrens bei der Erkennung von Leberkrankheiten und bösartigen Tumoren.

\footnotetext{
1) Die Arbeit enthält wesentliche Teile der Inauguraldissertation von $F$. W. Kolle.
} 


\section{Material und Methoden}

\section{Material}

Zur Orientierung über prinzipielle Unterschiede (Screening) bei der Bindung von Lectinen durch Proben Gesunder und Kranker untersuchten wir zwei Poolseren. Beide enthielten jeweils gleiche Anteile aus fünf Seren von Normalpersonen oder von Patienten, bei denen Lebermetastasen zu einer Cholestase geführt hatten (Tab. 3, Abb. 3). Die Abhängigkeit der Fällung von der Lectinkonzentration und der Inkubationszeit (Tab. 2, Abb. 1 u. 2) ermittelten wir mit elf zusätzlichen Seren: fünf stammten von Gesunden und je drei von Patienten mit alkoholinduzierter Lebercirrhose bzw. mit Lebermetastasen. Mit diesen 13 Proben verglichen wir außerdem die Fällung durch Concanavalin A mit der Affinitätschromatographie an Con A-Sepharose (7). Zuletzt prüften wir mit vier der zehn zum Screening eingesetzten Lectine die diagnostische Bedeutung der Reaktion. Hierzu wurden Seren von 80 Patienten mit histologisch gesicherten bösartigen oder Lebererkrankungen und von 20 Gesunden mit gleicher Altersverteilung untersucht (Tab. 4).

Lectine folgender Herkunft verwendeten wir ohne vorherige Reinigung: Canavalia ensiformis, Glycine max und Helix pomatia von Pharmacia (Uppsala), Dolichos biflorus und Ulex europaeus von Paesel (Frankfurt), Arachis hypogaea, Triticum vulgare, Ricinus communis 60 und 120 von Boehringer (Mannheim) sowie Phaseolus vulgaris (Typ S) von Serva (Heidelberg). $\alpha$-Methylmannosid bezogen wir von Ega-Chemie (Steinheim), Neuraminidase von Boehringer und von den Behringwerken (Marburg). Merck (Darmstadt) lieferte alle anderen Reagentien.

Wir setzten die Lösungen mit bidestilliertem demin. Wasser an. Lösung A enthielt zur Inkubation mit Neuraminidase (EC 3.2.1.18) Enzym aus $C l$. perfringens $300 \mathrm{U} / 1$ und aus $V$. cholerae 50000 Einh./1 (mod. nach 1.c. (8)). Bei den Fällungen wurden den Lösungen $B$ und $C$ Lectine zugesetzt.

Lösung A (Acetat-Tris $250 \mathrm{mmol} / 1$, Calciumchlorid $20 \mathrm{mmol} / 1$, pH 5,0)

Lösung B (Tris-Acetat $500 \mathrm{mmol} / \mathrm{l}$, Calciumchlorid $2 \mathrm{mmol} / \mathrm{l}$, Magnesiumchlorid $2 \mathrm{mmol} / \mathrm{l}, \mathrm{pH} 7,7$ )

Lösung C (Tris-Acetat $100 \mathrm{mmol} / \mathrm{l}$, Calciumchlorid $500 \mu \mathrm{mol} / \mathrm{l}$, Magnesiumchlorid $500 \mu \mathrm{mol} / 1$, Mangan(Il)chlorid $10 \mu \mathrm{mol} / \mathrm{l}, \mathrm{pH} 7,2)$

Geräte und Methoden

Mit Ausnahme der Apparatur zur Disk-Elektrophorese „Standard-PAA" von Biomol (Barth, Ilvesheim) benutzten wir Geräte von Eppendorf Gerätebau (Netheler \& Hinz, Hamburg).

Die Aktivität folgender Enzyme wurde bei $25^{\circ} \mathrm{C}$ im Mikroverfahren bestimmt:

Arylamidase, mikrosomal (EC 3.4.11.2) nach 1.c. (9), alkalische Phosphatase (EC 3.1.3.1) nach 1.c. (10), $\gamma$-Glutamyltransferase (EC 2.3.2.2) nach 1.c. (11), Cholinesterase (EC 3.1.1.8) nach 1.c. (12) und Arylesterase (EC 3.1.1.2) nach 1.c. (13).

Hierzu setzten wir $10 \mu \mathrm{l}$ (Cholinesterase) bis $100 \mu \mathrm{l}$ ( $\gamma$-Glutamyltransferase) Serum unmittelbar nach Abschluß der Blutgerinnung ein oder, ohne Abweichung in den Ergebnissen, nach Aufbewahrung bis zu $30 \mathrm{~d}$ bei $-28^{\circ} \mathrm{C}$ sofort nach dem Auftauen.

Bei der 2-Punkt-Bestimmung in suspendierten Niederschlägen wurden die Proben während der Inkubationszeit von $10 \mathrm{~min}$ (Cholinesterase) bis 30 min (Arylamidase, $\gamma$-Glutamyltransferase) geschüttelt und vor der Messung im Photometer $1101 \mathrm{M}$ zentrifugiert. Wir berücksichtigten die Eigenhy drolyse der Substrate und erhielten bei 20 Tests in Serie eine Reproduzierbarkeit von 4,9-5,7\% (Variationskoeffizient). Bei der Arylesterase-Bestimmung, wurde eine Präzision von 5,8\% erreicht, bei der kontinuierlichen Messung der übrigen Enzyme in Überständen, Kontrollen und gelösten Niederschlägen, registriert mit dem Kompensationsschreiber 4412, lagen die VK zwischen 2,9 und 3,5\%.

Wir inkubierten alle Ansätze bei $25^{\circ} \mathrm{C}$ in Reaktionsgefäßen 3810 , in denen anschließend Überstand und Präzipitat bei $12.000 \mathrm{gX}$ 2 min getrennt wurden. Der obigen Werten entsprechende VK für die Reproduzierbarkeit der Lectinfällung lag bei $7,6 \%$, ermittelt mit Cholinesterase aus Überständen nach Reaktion mit Concanavalin A $40 \mu \mathrm{mol} / 1$.
Nach Ermittlung der günstigsten Reaktionsbedingungen prüften wir in der ersten Versuchsreihe den Einfluß von N-Acetylneuraminsäureresten der Glykoenzyme auf ihre Lectinbindung mit folgender Anordnung:

\begin{tabular}{lllll} 
Ansatz*) & 1 & 2 & 3 & 4 \\
\hline Serum & $50 \mu \mathrm{l}$ & $50 \mu \mathrm{l}$ & $50 \mu \mathrm{l}$ & $50 \mu \mathrm{l}$ \\
Lösung A & $50 \mu \mathrm{l}$ & - & $50 \mu \mathrm{l}$ & - \\
Lsg. A + Neuraminidase - & $50 \mu \mathrm{l}$ & - & $50 \mu \mathrm{l}$ \\
\multicolumn{5}{c}{ Inkubation $16 \mathrm{~h}$ bei pH 5,5 } \\
Lösung B & $50 \mu \mathrm{l}$ & $50 \mu \mathrm{l}$ & - & - \\
Lsg. B + Lectin & - & - & $50 \mu \mathrm{l}$ & $50 \mu \mathrm{l}$ \\
& Inkubation 2 h bei pH 7,0 \\
\hline
\end{tabular}

\section{* 1 Sialoenzym ohne Hemmung (Kontrolle; Aktivität $=1,0$ ) \\ 2 Asialoenzym ohne Hemmung \\ 3 Lectinreaktion mit Sialoenzym \\ 4 Lectinreaktion mit Asialoenzym}

In einer zweiten Serie verzichteten wir auf die Vorbehandlung mit Neuraminidase und inkubierten, analog den Ansätzen 1 und 3, $100 \mu \mathrm{l}$ Serum mit $100 \mu \mathrm{l}$ Lösung C(mit/ohne Lectin) $2 \mathrm{~h}$ bei $25^{\circ} \mathrm{C}$. Nach Tests aus dem Überstand wurde der Niederschlag mit $500 \mu 1$ Lösung $C$ gewaschen. Ein Niederschlag des doppelten Ansatzes wurde mit dieser Lösung homogen suspendiert und auf ein mit dem Überstand identisches Volumen aufgefüllt. Das andere Präzipitat lösten wir auf das gleiche Volumen durch Anreiben mit Lösung C, die bindungsspezifische Zucker $0,5 \mathrm{~mol} / \mathrm{l}$ enthielt.

Qualitative Veränderungen der Enzyme nach Reaktion mit Lectinen untersuchten wir mit der Polyacrylamidgel-Elektrophorese. Dazu wurden Proben aus den Überständen deutlich gehemmter Ansätze in linearen Gradienten (14) unter folgenden Bedingungen getrennt: Polyacrylamid 36-216 g/1 Totalkonzentration, Röhrchen von $85 \mathrm{~mm}$ Länge und 4,8 $\mathrm{mm}$ lichter Weite, Rotationswinkel $5^{\circ}$, Gelpuffer: Tris-Schwefelsäure $1 \mathrm{~mol} / 1 \mathrm{pH} 7,2$, Elektrodenpuffer: Tris-Glycin $45 \mathrm{mmol} / 1 \mathrm{pH} 8,8$. Nach $18 \mathrm{~h}$ bei $40 \mathrm{~V}$ blieben alle Enzymfraktionen der Kontrollen noch frei beweglich, während Enzym-Lectin-Komplexe mit Molekulargewichten über 2,5 X $10^{6}$ Dalton vom weitporigen Gel am Start festgehalten wurden.

Die Zymogramme wurden wie folgt dargestellt: Arylamidase mit L-Alanyl-naphthyl-2-amid (15), alkalische Phosphatase mit Naphthyl-1-phosphat (16), $\gamma$-Glutamyltransferase mit 5-LGlutamylnaphthyl-2-amid $1,8 \mathrm{mmol} / 1, L$-Methionin $25 \mathrm{mmol} / 1$ und Glycylglycin $50 \mathrm{mmol} / 1 \mathrm{pH} 9,25$ (17, mod.), Cholinesterase und Arylesterase mit Naphthyl-2-acetat (18) in Gegenwart von Ethylendiamintetraacetat $20 \mathrm{mmol} / 1$ für Cholinesterase bzw. Paraoxon $1 \mu \mathrm{mol} / 1$ für Arylesterase. Vor der Aufbewahrung in Essigsäure 1,22 mol/1 $(70 \mathrm{ml} / \mathrm{l})$ entfärbten wir immer mit Dimethylformamid $0,72 \mathrm{~mol} / 1$ ( $50 \mathrm{ml} / \mathrm{l})$-Trichloressigsäure $1,22 \mathrm{~mol} / 1(200 \mathrm{~g} / \mathrm{l})$ mit Ausnahme der Proteinfärbung (14). Die Lokalisation der multiplen Formen bezogen wir auf die Laufstrecke des Albumins $\left(R_{F}=1,0\right)$. Die Reinheit der verwendeten Lectine wurde mit derselben Technik untersucht.

\section{Ergebnisse}

\section{Reaktionsbedingungen}

Das Lectin aus Dolichos biflorus enthielt pro g 13,6 U Arylamidase. Sie wanderte mit $R_{F} 0,85$ als anodische von sechs Proteinfraktionen, und ihr Anteil wurde von der Arylamidase-Aktivität im Test abgezogen. Die anderen Präparate waren katalytisch inaktiv, jedoch bis auf die Lectine aus Canavalia ensiformis, Glycine max, Helix pomatia und Triticum vulgare uneinheitlich. In der Lösung von Ulex europaeus fand sich nur das Isolectin I (Tab. 1). 
Tab. 1. Eigenschaften der verwendeten Lectine. Fraktionierung durch Disk-Elektrophorese (14).

\begin{tabular}{|c|c|c|c|c|}
\hline Herkunft & Molekulargewicht & Referenz & Spezifität für Zucker & Gehalt, Fraktionen \\
\hline $\begin{array}{l}\text { Arachis hypogaea } \\
\text { (Erdnuß) }\end{array}$ & $\begin{array}{l}108.000 * \\
120.000\end{array}$ & $\begin{array}{l}(1) \\
\text { (Lieferant) }\end{array}$ & Galaktose & $\sim 98 \%, 3$ Banden \\
\hline $\begin{array}{l}\text { Canavalia ensiformis } \\
\text { (Schwertbohne) }\end{array}$ & $\begin{array}{l}55.000^{*} \\
51.000\end{array}$ & $\begin{array}{l}(1) \\
\text { (Lieferant) }\end{array}$ & Mannose, Glucose & $>98 \%, 1$ Bande \\
\hline $\begin{array}{l}\text { Dolichos biflorus } \\
\text { (Helmbohne) }\end{array}$ & $\begin{array}{l}140.000^{*} \\
124.000\end{array}$ & $\begin{array}{l}(1) \\
(19)\end{array}$ & N-Acetylgalaktosamin & $\sim 50 \%, 6$ Banden \\
\hline $\begin{array}{l}\text { Glycine max } \\
\text { (Sojabohne) }\end{array}$ & $120.000 *$ & (Lieferant) & $\begin{array}{l}\text { Galaktose, } \\
\text { N-Acetylgalaktosamin }\end{array}$ & $99 \%, 1$ Bande \\
\hline $\begin{array}{l}\text { Helix pomatia } \\
\text { (Weinbergschnecke) }\end{array}$ & $79.000^{*}$ & (Lieferant) & $\mathrm{N}$-Acetylgalaktosamin & $\sim 95 \%, 1$ Bande \\
\hline $\begin{array}{l}\text { Phaseolus vulgaris } \\
\text { (Feuerbohne) }\end{array}$ & $128.000 *$ Typ S & (1) & $\begin{array}{l}\text { Galaktose, } \\
\text { N-Acetylgalaktosamin }\end{array}$ & $\sim 75 \%, 4$ Banden \\
\hline $\begin{array}{l}\text { Ricinus communis } \\
\text { (Castorbohne) }\end{array}$ & $\begin{array}{l}60.000 * \text { Typ I } \\
120.000 *\end{array}$ & $\begin{array}{l}\text { (Lieferant) } \\
\text { (Lieferant) }\end{array}$ & $\begin{array}{l}\text { Galaktose } \\
\text { Galaktose }\end{array}$ & $\begin{array}{l}\sim 95 \%, 3 \text { Banden } \\
\sim 95 \%, 3 \text { Banden }\end{array}$ \\
\hline $\begin{array}{l}\text { Triticum vulgare } \\
\text { (Weizen) }\end{array}$ & $36.000^{*}$ & (Lieferant) & N-Acetylglucosamin & $>98 \%, 1$ Bande \\
\hline $\begin{array}{l}\text { Ulex europaeus } \\
\text { (Stechginster) }\end{array}$ & $\begin{array}{l}46.000 * \text { Typ I } \\
170.000 \text { Typ II }\end{array}$ & $\begin{array}{l}(1) \\
\text { (Lieferant) }\end{array}$ & $\begin{array}{l}\text { Fucose } \\
\text { N-Acetylglucosamin }\end{array}$ & $\begin{array}{l}\sim 95 \%, 3 \text { Banden } \\
\text { Spur }\end{array}$ \\
\hline
\end{tabular}

*liegen den Konzentrationsangaben zugrunde

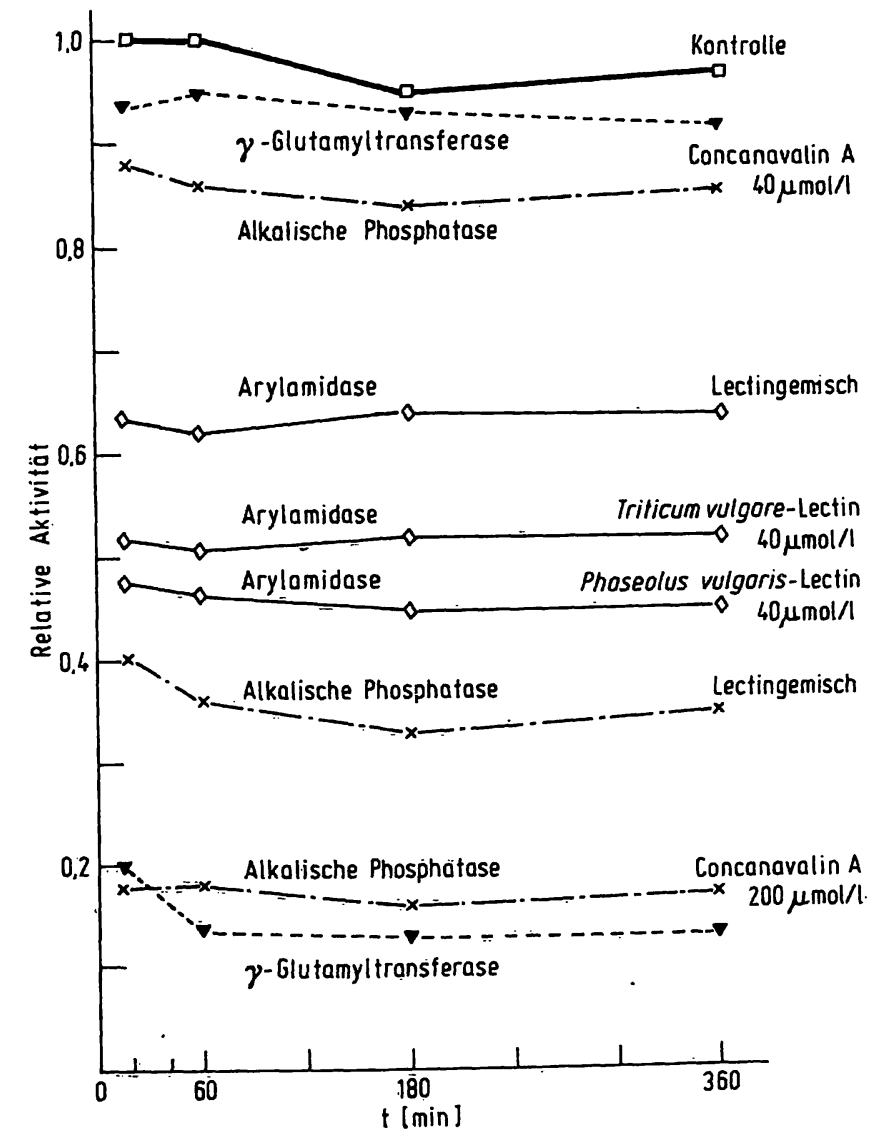

Abb. 1. Relative Aktivität (Ordinate) von Arylamidase, alkalischer Phosphatase und $\gamma$-Glutamyltransferase im Überstand eines gefällten Poolserums bei zunehmender Inkubationsdauer (Absżisse) mit verschiedenen Lectinen. Lectingemisch: Canavalia ensiformis $20 \mu \mathrm{mol} / 1$, Triticum vulgaré $20 \mu \mathrm{mol} / 1$, Glycine max $12 \mu \mathrm{mol} / 1$, Ricinus communisAgglutinin $12010 \mu \mathrm{mol} / 1$. Vorgehen s. Methodik.
Den Untersuchungen von Köttgen \& Gerok (4) mit Concanavalin A entsprechend fällten in unseren Ansätzen mit Ausnahme der Lectine aus Glycine max und Helix pomatia alle anderen innerhalb von Minuten. Nach 60 min war die Reaktion nahezu abgeschlossen, so daß mit der gewählten Inkubationszeit von $2 \mathrm{~h}$ auch verzögerte Präzipitationen erfaßt wurden (Abb. 1). Zwischen 15 und $37^{\circ} \mathrm{C}$ hatte die Temperatur keinen Einfluß auf die Fällung, die mit steigender Lectinkonzentration zunahm. So war Concanavalin A bei $20 \mu \mathrm{mol} / \mathrm{l}$ praktisch unwirksam, bei $40 \mu \mathrm{mol} / 1$ und $66 \mu \mathrm{mol} / 1$ (5) wurden Cholinesterase und alkalische Phosphatase deutlich gefällt und bei $182 \mu \mathrm{mol} / 1$ (4) und $200 \mu \mathrm{mol} / 1$ ließen sich nur noch $\gamma$-Glutamyltransferase und Arylesterase mit Restaktivitäten von mehr als 0,40 im Überstand messen (Abb. 2). Höhere Konzentrationen bis $500 \mu \mathrm{mol} / \mathrm{l}$ führten zu einer stärkeren Präzipitation. Bei Lectin aus Triticum vulgare erreichten wir mit $200 \mu \mathrm{mol} / 1$, aus Phaseolus vulgaris mit $100 \mu \mathrm{mol} / 1$ den Endpunkt.

Die Abnahme der Aktivität im Überstand folgte dabei in allen Seren trotz unterschiedlicher Restaktivität einer offenbar sigmoidalen Kurve (Abb. 2). Damit war das Verhältnis der Restaktivitäten bei zwei Lectinkonzentrationen, ausgedrückt als Quotient, wegen der gleichartigen Kurvenneigung konstant und für jedes Enzym typisch. So wurde mit 13 Seren aus der Reaktion mit Concanavalin A bei $200 \mu \mathrm{mol} / \mathrm{l}$ und $40 \mu \mathrm{mol} / 1$ für die alkalische Phosphatase ein Quotient von 0,19 0,03 $(\bar{x} \pm s)$ und für $\gamma$-Glutamyltransferase von $0,27 \pm 0,04$ errechnet. Wegen dieses gleichartigen Verhaltens ist im Prinzip jede effektive Lectinkonzentration zur Differenzierung geeignet, da die untersuchten Seren bei verschiedenen Konzentrationen des Agglutinins dieselben Ab- 


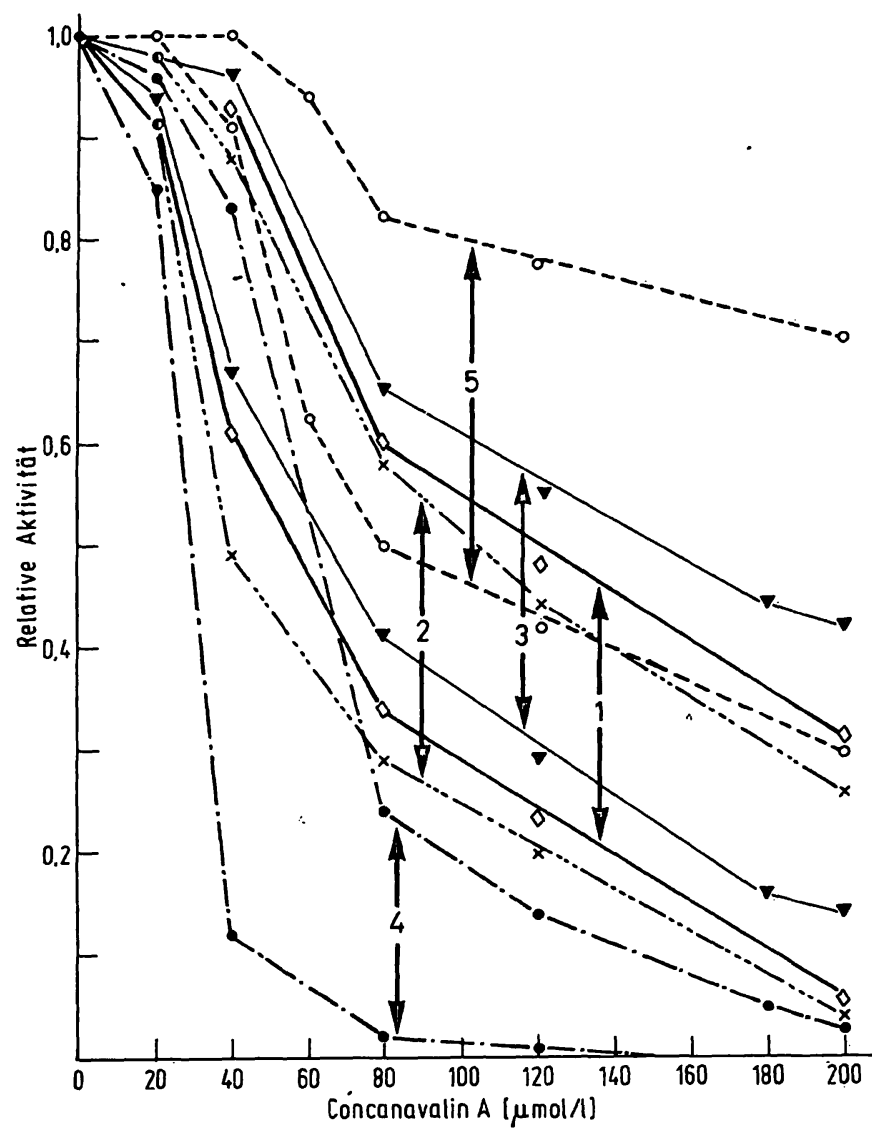

Abb. 2. Relative Aktivität (Ordinate) von fünf Enzy men in den Überständen von 13 Seren nach Reaktion mit Concanavalin A-Lösungen steigender Konzentration (Abszisse).

Die Pfeile bezeichnen die Variationsbreite in diesem Kollektiv. Inkubationszeit $2 \mathrm{~h}$. Vorgehen s. Methodik.

\begin{tabular}{|c|c|}
\hline $\begin{array}{l}\text { Arylamidase } \\
\text { Alkalische Phosphatase } \\
\gamma \text {-Glutamyltransferase }\end{array}$ & 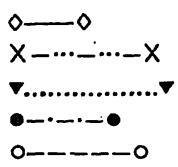 \\
\hline
\end{tabular}

weichungen voneinander zeigten. Dennoch wählten wir, soweit es die Löslichkeit der Lectine erlaubte, Konzentrationen zwischen 20 und $50 \mu \mathrm{mol} / 1$, da hier noch gut meßbare Aktivitäten im Überstand vorlagen. Wie bei Concanavalin A nahm auch die Präzipitation durch andere Lectine in der Reihenfolge Arylesterase $-\gamma$ Glutamyltransferase - Arylamidase - alkalische Phosphatase - Cholinesterase zu (Abb. 2).

Die Selektivität der Fällung ergab sich aus ihrer unterschiedlichen Hemmung durch Zugabe von bindungsspezifischen Zuckern in zweihundertfachem Überschuß, bezogen auf das Agglutinin. Während Galaktose und NAcetylgalaktosamin die Präzipitation von Arylamidase durch Phaseolus vulgaris-Lectin $20 \mu \mathrm{mol} / 1$ nicht veränderten, hemmte N-Acetylglucosamin die Reaktion von Triticum vulgare-Agglutinin $40 \mu \mathrm{mol} / 1 \mathrm{mit}$ Arylamidase, alkalischer Phosphatase und.Cholinesterase komplett. Glucose war bei der Interaktion von Concanavalin A $180 \mu \mathrm{mol} / 1 \mathrm{mit}$ Cholinesterase unwirksam, doch erhöhte sie die Restaktivität der $\gamma$-Glutamyltransferase von $0,25 \pm 0,06$ auf 0,52 $\pm 0,08$. Bei derselben Konzentration von Concanavalin A wirkte $\alpha$-Methylmannosid wie Glucose auf die Bindung von $\gamma$-Glutamyltransferase, und es blockierte die Fällung von Cholinesterase sogar vollständig.

\section{Screening und diagnostische Bedeutung}

Messungen im Überstand und im suspendierten und gelösten Niederschlag ließen eine verlustfreie Fällung annehmen, da die Aktivität des präzipitierten Anteils zumeist der Differenz der katalytischen Konzentration von Serum und Überstand entsprach (Tab. 2). Danach war der Lectinrezeptor des Enzyms offenbar nicht in der Nähẹ des aktiven Zentrums gelegen.

Tab. 2. Aktivitätsverteilung bei Fällung von Enzymen durch Lectine verschiedenen Ursprụngs. Angabe der relativen katalytischen Konzentration im Überstand (obere Zahlen) und im suspendierten Niederschlag. Ursprüngliche Aktivität des Serums $=1,0$. Vorgehen s. Methodik, *nicht gemessen.

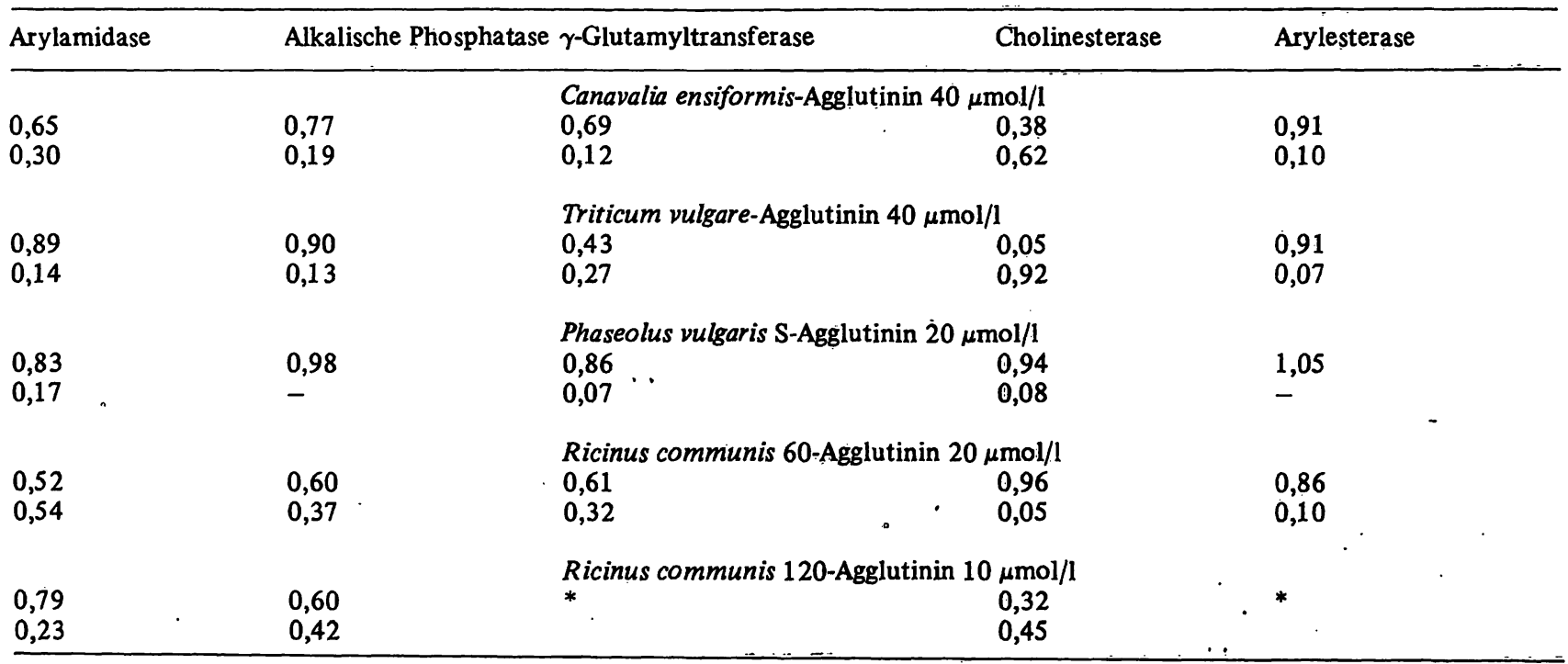


Gemessen am Volumen des Niederschlages fällten Concanavalin A und die Lectine aus Triticum vulgare und Phaseolus vulgaris besonders stark. Arylesterase ausgenommen, entsprach das Verhalten der Enzyme dieser sichtbaren Präzipitation, die mit den Lectinen aus Dolichos biflorus, Glycine max, Helix pomatia und Ulex europaeus gering ausfiel (Tab. 3). Die Vorbehandlung mit Neuraminidase verstärkte die Fällung durch Glycine max-Agglutinin, und nur bei Cholinesterase fuihrte die Abspaltung von N-Acetylneuraminsäure zu besserer Bindung mit anderen Lectinen. Da sie die Unterscheidung zwischen den Seren Gesunder und Krebskranker nicht verbesserte, wurde auf sie verzichtet.

Vielmehr differierten die Poolseren am deutlichsten bei der Reaktion von Arylamidase mit den Lectinen aus Phaseolus vulgaris und Triticum vulgare, bei der Fällung der alkalischen Phosphatase durch Triticum vulgare- und Ulex europaeus-Agglutinin und bei der Bindung von Cholinesterase mit Concanavalin A. Daher wählten wir diese fünf Kombinationen für die systematische Untersuchung von Seren bei verschiedenen Erkrankungen aus. Hinzu nahmen wir die Reaktion von Arylesterase mit
Triticum vulgare-Lectin und zum Vergleich, da bereits von anderer Seite untersucht (4), die Hemmung der $\gamma$-Glutamyltransferase durch Concanavalin A (Tab. 4), obwohl dieses Enzym weder bei der Fällung mit 200 $\mu \mathrm{mol} / \mathrm{l}$, noch bei der Adsorption an Con A-Sepharose (7) Differenzen zwischen den Seren Gesunder und Kranker erkennen ließ: Im Úberstand lag die Restaktivität bei fünf gesunden Probanden um 0,22 (0,20-0,24), bei den drei Patienten mit Lebercirrhose zwischen 0,18 und 0,28 und den drei Kranken mit Lebermetastasen zwischen 0,16 und 0,22. Im Aufgabeeluat der Con A-SepharoseSäule betrugen die entsprechenden Anteile der N-Acetylneuraminsäure enthaltenden (fetalen) $\boldsymbol{\gamma}$-Glutamyltransferase $0,38(0,30-0,47)$ bzw. $0,24-0,31$ und $0,23-0,39$. Somit war im Gegensatz zu den Angaben von Köttgen et al. (20) eine sichere Unterscheidung zwischen den Seren Gesunder und Leberkranker nicht zu erreichen.

Dies gelang dagegen mit der Hemmung von Arylamidase durch Phaseolus vulgaris-Agglutinin (Tab. 4). Ähnlich effektiv waren, bis auf die Erkennung chronischer Hepatitiden, die Fällung von Arylamidase durch Triticum vulgare-Lectin, von alkalischer Phosphatase durch Ulex

Tab. 3. Relative Aktivität in den Überständen von Poolseren Gesunder (G) und Kranker mit Lebermetastasen (C) nach Fällung mit Lectinen, bezogen auf die katalytische Konzentration im Serum ohne (jeweils obere Reihe) oder nach (jeweils untere Reihe) Vorbehandlung mit Neuraminidase (Ausgangsaktivität jeweils 1,0). Beurteilung der sichtbaren Fällung: 0 keinc, $(+)$ schwache, + deutliche, ++ starke Reaktion. Mittel aus 2 Doppelbestimmungen, Vorgehen s. Methodik.

\begin{tabular}{|c|c|c|c|c|c|c|c|c|c|c|c|c|c|}
\hline & & & & \multicolumn{2}{|c|}{ Arylamidase } & \multicolumn{2}{|c|}{$\begin{array}{l}\text { Alkalische } \\
\text { Phosphatase }\end{array}$} & \multicolumn{2}{|c|}{$\begin{array}{l}\gamma \text {-Glutamyl- } \\
\text { transferase }\end{array}$} & \multicolumn{2}{|c|}{$\begin{array}{l}\text { Cholin- } \\
\text { esterase }\end{array}$} & \multicolumn{2}{|c|}{ Arylesterase } \\
\hline & & & & G & $\mathrm{C}$ & G & $\mathrm{C}$ & G & C & G & $\mathrm{C}$ & G & $\mathrm{C}$ \\
\hline & & & & $\mathrm{U} / 1$ & & $\mathrm{U} / 1$ & & $\mathrm{U} / 1$ & & $\mathrm{U} / 1$ & & $\mathrm{kU} / \mathrm{l}$ & \\
\hline \multicolumn{4}{|c|}{$\begin{array}{l}\text { Aktivität im Serum ohne Neuraminidase } \\
\text { nach Neuraminidase }\end{array}$} & $\begin{array}{l}34,5 \\
32,4\end{array}$ & $\begin{array}{l}105 \\
105\end{array}$ & $\begin{array}{l}108 \\
120\end{array}$ & $\begin{array}{l}529 \\
585\end{array}$ & $\begin{array}{l}19,8 \\
17,6\end{array}$ & $\begin{array}{l}236 \\
198\end{array}$ & $\begin{array}{l}4810 \\
5100\end{array}$ & $\begin{array}{l}3760 \\
3470\end{array}$ & $\begin{array}{l}155 \\
160\end{array}$ & $\begin{array}{l}84,0 \\
80,5\end{array}$ \\
\hline \multicolumn{14}{|c|}{ Lectin } \\
\hline \multirow[t]{2}{*}{ Herkunft } & \multirow{2}{*}{$\begin{array}{l}\text { Konzentra- } \\
\text { tion } \\
(\mu \mathrm{mol} / 1)\end{array}$} & Fällu & & \multirow{2}{*}{\multicolumn{6}{|c|}{ Relative Aktivität im Überstand }} & & & & \\
\hline & & G & $\mathrm{C}$ & & & & & & & & & & \\
\hline Arachis hypogaea & 10 & $\begin{array}{l}(+) \\
(+)\end{array}$ & $\begin{array}{l}(+) \\
(+)\end{array}$ & $\begin{array}{l}0,88 \\
0,90\end{array}$ & $\begin{array}{l}0,78 \\
0,80\end{array}$ & $\begin{array}{l}0,88 \\
0,84\end{array}$ & $\begin{array}{l}0,90 \\
0,90\end{array}$ & $\begin{array}{l}0,73 \\
0,78\end{array}$ & $\begin{array}{l}0,81 \\
0,85\end{array}$ & $\begin{array}{l}0,82 \\
0,72\end{array}$ & $\begin{array}{l}0,81 \\
0,85\end{array}$ & $\begin{array}{l}1,00 \\
0,77\end{array}$ & $\begin{array}{l}1,00 \\
0,67\end{array}$ \\
\hline \multirow[t]{2}{*}{ Canavaliạ enșiformis } & 40 & $\begin{array}{l}+ \\
+\end{array}$ & $\begin{array}{l}++ \\
+\end{array}$ & $\begin{array}{l}0,61 \\
0,62\end{array}$ & $\begin{array}{l}0,66 \\
0,58\end{array}$ & $\begin{array}{l}0,49 \\
0,66\end{array}$ & $\begin{array}{l}0,54 \\
0,76\end{array}$ & $\begin{array}{l}0,80 \\
0,63\end{array}$ & $\begin{array}{l}0,84 \\
0,57\end{array}$ & $\begin{array}{l}0,10 \\
0,05\end{array}$ & $\begin{array}{l}0,38 \\
0,29\end{array}$ & $\begin{array}{l}0,95 \\
0,73\end{array}$ & $\begin{array}{l}0,78 \\
0,78\end{array}$ \\
\hline & 200 & $\begin{array}{l}++ \\
+\end{array}$ & $\begin{array}{l}+ \\
+\end{array}$ & $\begin{array}{l}0,30 \\
0,27\end{array}$ & $\begin{array}{l}0,13 \\
0,12\end{array}$ & $\begin{array}{l}0 ; 16 \\
0,14\end{array}$ & $\begin{array}{l}0,08 \\
0,07\end{array}$ & $\begin{array}{l}0,32 \\
0,33\end{array}$ & $\begin{array}{l}0,13 \\
0,10\end{array}$ & $\begin{array}{l}0,03 \\
0,02\end{array}$ & $\begin{array}{l}0,01 \\
0,01\end{array}$ & $\begin{array}{l}0,30 \\
0,30\end{array}$ & $\begin{array}{l}0,28 \\
0,28\end{array}$ \\
\hline Dolichos biflorus & 20 & $\begin{array}{l}(+) \\
(+)\end{array}$ & $\begin{array}{l}(+) \\
(+)\end{array}$ & $\begin{array}{l}1,00 \\
1,05\end{array}$ & $\begin{array}{l}1,00 \\
1,00\end{array}$ & $\begin{array}{l}0,93 \\
0.87\end{array}$ & $\begin{array}{l}0,98 \\
0,98\end{array}$ & $\begin{array}{l}0,94 \\
0,91\end{array}$ & $\begin{array}{l}1,20 \\
1,15\end{array}$ & $\begin{array}{l}0,94 \\
0,90\end{array}$ & $\begin{array}{l}0,90 \\
1,00\end{array}$ & $\begin{array}{l}1,00 \\
1,06\end{array}$ & $\begin{array}{l}1,00 \\
0,90\end{array}$ \\
\hline Glycine max & 40 & $\begin{array}{l}0 \\
+\end{array}$ & $\begin{array}{l}0 \\
+\end{array}$ & $\begin{array}{l}0,98 \\
0,60\end{array}$ & $\begin{array}{l}1,05 \\
0,73\end{array}$ & $\begin{array}{l}1,00 \\
0,40\end{array}$ & $\begin{array}{l}0,83 \\
0,46\end{array}$ & $\begin{array}{l}0,95 \\
0,70\end{array}$ & $\begin{array}{l}0,90 \\
0,50\end{array}$ & $\begin{array}{l}0,87 \\
0,03\end{array}$ & $\begin{array}{l}1,00 \\
0,05\end{array}$ & $\begin{array}{l}1,00 \\
1,07\end{array}$ & $\begin{array}{l}1,00 \\
0,88\end{array}$ \\
\hline Helix pomatia & 40 & $\begin{array}{l}0 \\
(+)\end{array}$ & $\begin{array}{l}0 \\
(+)\end{array}$ & $\begin{array}{l}0,92 \\
0,94\end{array}$ & $\begin{array}{l}1,02 \\
0,90\end{array}$ & $\begin{array}{l}0,86 \\
0,91\end{array}$ & $\begin{array}{r}\cdot 0,92 \\
0,99\end{array}$ & $\begin{array}{l}0,93 \\
0,90\end{array}$ & $\begin{array}{l}1,20 \\
1,10\end{array}$ & $\begin{array}{l}0,93 \\
0,90\end{array}$ & $\begin{array}{l}0,82 \\
0,90\end{array}$ & $\begin{array}{l}1,03 \\
1,10\end{array}$ & $\begin{array}{l}1,02 \\
0,93\end{array}$ \\
\hline Phaseolus vulgaris S & 20 & $\begin{array}{l}++ \\
++\end{array}$ & $\begin{array}{l}++ \\
++\end{array}$ & $\begin{array}{l}0,30 \\
0,61\end{array}$ & $\begin{array}{l}0,76 \\
0,65\end{array}$ & $\begin{array}{l}0,61 \\
0,83\end{array}$ & $\begin{array}{l}0,90 \\
0,82\end{array}$ & $\begin{array}{l}0,85 \\
0,82\end{array}$ & $\begin{array}{l}0,84 \\
0,69\end{array}$ & $\begin{array}{l}0,81 \\
0,24\end{array}$ & $\begin{array}{l}0,78 \\
0,44\end{array}$ & $\begin{array}{l}1,06 \\
1,02\end{array}$ & $\begin{array}{l}0,90 \\
0,76\end{array}$ \\
\hline Ricinus communis 60 & 20 & $\begin{array}{l}(+) \\
+\end{array}$ & + & $\begin{array}{l}1,01 \\
0,98\end{array}$ & $\begin{array}{l}0,95 \\
0,83\end{array}$ & $\begin{array}{l}0,71 \\
0,69\end{array}$ & $\begin{array}{l}0,57 \\
0,73\end{array}$ & $\begin{array}{l}0,91 \\
0,97\end{array}$ & $\begin{array}{l}0,90 \\
0,75\end{array}$ & $\begin{array}{l}0,84 \\
0,34\end{array}$ & $\begin{array}{l}1,00 \\
0,49\end{array}$ & $\begin{array}{l}1,10 \\
1,00\end{array}$ & $\begin{array}{l}0,90 \\
0,84\end{array}$ \\
\hline Ricinus communis 120 & 10 & $\begin{array}{l}+ \\
+\end{array}$ & + & $\begin{array}{l}0,86 \\
0,75\end{array}$ & $\begin{array}{l}0,79 \\
0,81\end{array}$ & $\begin{array}{l}0,64 \\
0,60\end{array}$ & $\begin{array}{l}0,53 \\
0,63\end{array}$ & $\begin{array}{l}0,93 \\
0,99\end{array}$ & $\begin{array}{l}0,77 \\
0,70\end{array}$ & $\begin{array}{l}0,44 \\
0,07\end{array}$ & $\begin{array}{l}0,54 \\
0,24\end{array}$ & $\begin{array}{l}1,03 \\
0,98\end{array}$ & $\begin{array}{l}0.86 \\
0,97\end{array}$ \\
\hline Triticum vulgare & 40 & $\begin{array}{l}++ \\
++\end{array}$ & $\begin{array}{l}++ \\
+\end{array}$ & $\begin{array}{l}0,45 \\
0,80\end{array}$ & $\begin{array}{l}0,77 \\
0,98\end{array}$ & $\begin{array}{l}0,58 \\
1,15\end{array}$ & $\begin{array}{l}0,82 \\
1,00\end{array}$ & $\begin{array}{l}0,77 \\
0,93\end{array}$ & $\begin{array}{l}0,74 \\
0,87\end{array}$ & $\begin{array}{l}0,72 \\
0,95\end{array}$ & $\begin{array}{l}0,80 \\
0,98\end{array}$ & $\begin{array}{l}1,04 \\
1,08\end{array}$ & $\begin{array}{l}0,90 \\
1,05\end{array}$ \\
\hline Ulex europaeus & 150 & $\begin{array}{l}(+) \\
+\end{array}$ & $\begin{array}{l}(+) \\
+\end{array}$ & $\begin{array}{l}1,00 \\
1,07\end{array}$ & $\begin{array}{l}1,00 \\
0,99\end{array}$ & $\begin{array}{l}0,82 \\
0,94\end{array}$ & $\begin{array}{l}1,02 \\
1,02\end{array}$ & $\begin{array}{l}0,96 \\
0,88\end{array}$ & $\begin{array}{l}1,12 \\
1,10\end{array}$ & $\begin{array}{l}0,96 \\
0,92\end{array}$ & $\begin{array}{l}0,90 \\
0,98\end{array}$ & $\begin{array}{l}0,94 \\
1,01\end{array}$ & $\begin{array}{l}1,06 \\
0,98\end{array}$ \\
\hline
\end{tabular}


Tab. 4. Aktivität mikrosomaler Enzyme in Seren und in Uberständen nach Fällung mit Lectinen.

Angabe der katalytischen Konzentration im Serum (relative Aktivität 1,0) als Bereich mit Grenzwerten, im Überstand als relative Aktivität (Mittelwert \pm Standardabweichung). Vorgehen s. Methodik. - Berechnung signifikanter Abweichungen vom Normalkollektiv mit dem Wilcoxon-Rangsummen-Test für nicht korrelierte Stichproben: ${ }^{*} p<0,01,{ }^{\bullet}<0,1$
A. Gesunde Probanden, $\mathrm{n}=20$
B. Chronische Hepatitis, $n=10$ (Chron. persistierende H. 8, chron. aggressive H. 2)
C. Alkoholinduzierte Fettleber, $n=17$ (mit entzündlicher Aktivität 12, ohne Entzündung 5)
D. Lebercirrhose, $n=15$ (mit entzündlicher Aktivität 4, ohne Zeichen einer Entzündung 11)
E. Tumoren ohne Metastasen in der Leber, $n=20$ (Bronchus 6, Mamma 10, Colon 4)
F. Tumoren mit Lebermetastasen, $n=17$ (Bronchus 6, Mamma 4, Colon 4, Magen 3)

$\begin{array}{llllll}\text { A } & \text { B } & \text { C } & \text { D } & \text { E } & \text { F }\end{array}$

Enzym und Herkunft des fällenden Lectins

\begin{tabular}{lcrcccccc}
\hline Arylamidase & $(\mathrm{U} / \mathrm{l})$ & $13-43$ & $29-152$ & $30-94$ & $22-52$ & $23-145$ & $22-255$ \\
Triticum vulgare & $40 \mu \mathrm{mol} / 1$ & $0,40 \pm 0,15$ & $0,52 \pm 0,18^{\bullet}$ & $0,61 \pm 0,11^{*}$ & $0,55 \pm 0,16^{*}$ & $0,61 \pm 0,16^{*}$ & $0,70 \pm 0,12^{*}$ \\
Phaseolus vulgaris $\mathrm{S}$ & $20 \mu \mathrm{mol} / 1$ & $0,25 \pm 0,13$ & $0,43 \pm 0,16^{*}$ & $0,43 \pm 0,16^{*}$ & $0,42 \pm 0,12^{*}$ & $0,51 \pm 0,21^{*}$ & $0,64 \pm 0,20^{*}$ \\
Alkalische Phosphatase & $(\mathrm{U} / \mathrm{l})$ & $42-98$ & $58-495$ & $48-152$ & $67-293$ & $65-1129$ & $90-1532$ \\
Triticum vulgare & $40 \mu \mathrm{mol} / 1$ & $0,60 \pm 0,16$ & $0,54 \pm 0,17$ & $0,59 \pm 0,13$ & $0,61 \pm 0,22$ & $0,58 \pm 0,23$ & $0,68 \pm 0,17$ \\
Ulex europaeus & $150 \mu \mathrm{mol} / 1$ & $0,78 \pm 0,16$ & $1,03 \pm 0,14^{*}$ & $1,10 \pm 0,19^{*}$ & $1,07 \pm 0,21^{*}$ & $1,00^{*} \pm 0,17^{*}$ & $1,07 \pm 0,18^{*}$ \\
$\gamma$-Glutamyltraneferase & $(\mathrm{U} / \mathrm{l})$ & $7-40$ & $10-176$ & $14 \pm 590$ & $18-146$ & $7-347$ & $10 \pm 395$ \\
Canavalia ensiformis & $40 \mu \mathrm{mol} / 1$ & $0,77 \pm 0,11$ & $0,73 \pm 0,11$ & $0,83 \pm 0,17$ & $0,81 \pm 0,11$ & $0,81 \pm 0,15$ & $0,71 \pm 0,13$ \\
Cholinesterase & $(\mathrm{U} / \mathrm{l})$ & $3010-6300$ & $3030-7020$ & $2050-6510$ & $1000-5040$ & $2050-5080$ & $1140-3700$ \\
Canavalia ensiformis & $40 \mu \mathrm{mol} / 1$ & $0,10 \pm 0,05$ & $0,13 \pm 0,06^{\bullet}$ & $0,21 \pm 0,10^{*}$ & $0,15 \pm 0,08^{*}$ & $0,38 \pm 0,26^{*}$ & $0,38 \pm 0,19 *$ \\
Arylesterase & $(\mathrm{kU} / \mathrm{l})$ & $55-204$ & $51-143$ & $41-165$ & $20-100$ & $46-111$ & $37-133$ \\
Triticum vulgare & $40 \mu \mathrm{mol} / 1$ & $0,91 \pm 0,13$ & $0,95 \pm 0,17$ & $0,99 \pm 0,17$ & $1,08 \pm 0,17$ & $1,06 \pm 0,14$ & $1,06 \pm 0,15$ \\
& & & & & & & &
\end{tabular}

europaeus-Lectin und von Cholinesterase durch Concanavalin A. Die größten Abweichungen vom Normalverhalten fanden sich mit allen Kombinationen erwartungsgemäß beim Vorliegen von Lebermetastasen. Ebenso einheitlich zeigten die Enzyme bei Tumorleiden und Leberkrankheiten eine gegenüber der Norm erhöhte Lectinresistenz, wobei Arylesterase durch Triticum vulgare-Lectin und alkalische Phosphatase durch Ulex europaeus-Lectin in ihrer Aktivität sogar stimuliert wurden (Tab. 4).

\section{Multiple Formen}

Die eingesetzte Lectinkonzentration bestimmte auch die Verteilung der Enzymaktivität des Überstandes bei der Elektrophorese im Poly acrylamidgel. Da Concanavalin A $200 \mu \mathrm{mol} / 1$ in den Zymogrammen alle Fraktionen bis auf die anodisch gelegene Hauptbande eliminierte, benutzten wir eine Konzentration von $60 \mu \mathrm{mol} / \mathrm{l}$. Phaseolus vulgaris-Agglutinin verwendeten wir mit $40 \mu \mathrm{mol} / 1$, bei Ricinus communis-Agglutinin 120 wählten wir $20 \mu \mathrm{mol} / 1$ und im übrigen die Ansätze von Tabelle 3. Unter diesen Bedingungen veränderte sich die einzige Fraktion der alkalischen Phosphatase nicht. Während bei Arylamidase die einheitliche, anodisch lokalisierte Aktivität im Serum Gesunder durch die Einwirkung von Lectinen lediglich abnahm, traten in den übrigen Überständen komplexe Muster auf (Abb. 3): Bei Krebskranken mit Cholestase zeigten sich 5 Arylamidase-Fraktionen, die Anfärbung des kathodischen Gelendes eingeschlossen. Ricinus communisAgglutinin 120 und Phaseolus vulgaris-Agglutinin hatten keinen Einfluß auf diese Verteilung, doch löschten Triticum vulgare-Lectin und Concanavalin $\mathrm{A}$ die vierte Bande und verringerten die Intensität der ersten und dritten (Abb. 4).
Die Darstellung von Isoenzymen der $\gamma$-Glutamyltransferase nâch der Literatur $(17,21,22,23)$ ist nicht absolut spezifisch, da Naphthyl-2-amin und 4-Nitroanilin während der langen Inkubation auch durch Arylamidase freigesetzt werden. Dies erklärt ältere Mitteilungen über die identische Lokalisation von $\gamma$-Glutamyltransferase- und Arylamidase-Fraktionen (24) und erfordert eine Hemmung von Arylamidase durch $L$-Methionin. In normalaktiven Seren waren die Banden der $\gamma$-Glutamyltransferase allerdings so schwach, daß ihre Beurteilung nach der Reaktion mit Lectinen unmöglich war. Im Poolserum der Malignomträger ließen sich die anodische Hauptfraktion und die Aktivität am Gelanfang auch mit Concanavalin A, das die Muster aller untersuchten Enzyme am stärksten reduzierte, nicht fällen (Abb. 3 und 4). Dagegen minderten Triticum vulgare-Lectin und - schwächer - Ricinus communis 60 die Darstellung der langsam wandernden $\left(\mathrm{R}_{\mathrm{F}} 0,28\right)$ Bande. Ricinus communis-Agglutinin 120 veränderte keine der 4 Fraktionen.

Zum Nachweis multipler Formen der Cholinesterase war Naphthyl-2-acetat (18), mit dem wir die Ergebnisse von Gaffney (25) bestätigten, dem langsam hydrolysierenden Essigsäure-3-indolylester (26) überlegen. Seine Spaltung übertraf auch die von Butyrylthiocholin (27), da, trotz Zusatz von Paraoxon oder Diisopropylfluorphosphat, freigesetztes Thiocholin und Sulfhydrylgruppen der Proteine durch Darstellung mit Schwermetallionen nicht sicher zu unterscheiden waren. Dagegen trat bei Inkubation von Cholinesterase und Arylesterase mit Naphthyl2-acetat immer eine gegen Lectinfällung resistente Fraktion auf. Sie erschien in der Position von Albumin $\left(R_{\mathrm{F}} 1,0\right)$ bei dessen Markierung durch Fluorescein (28) 


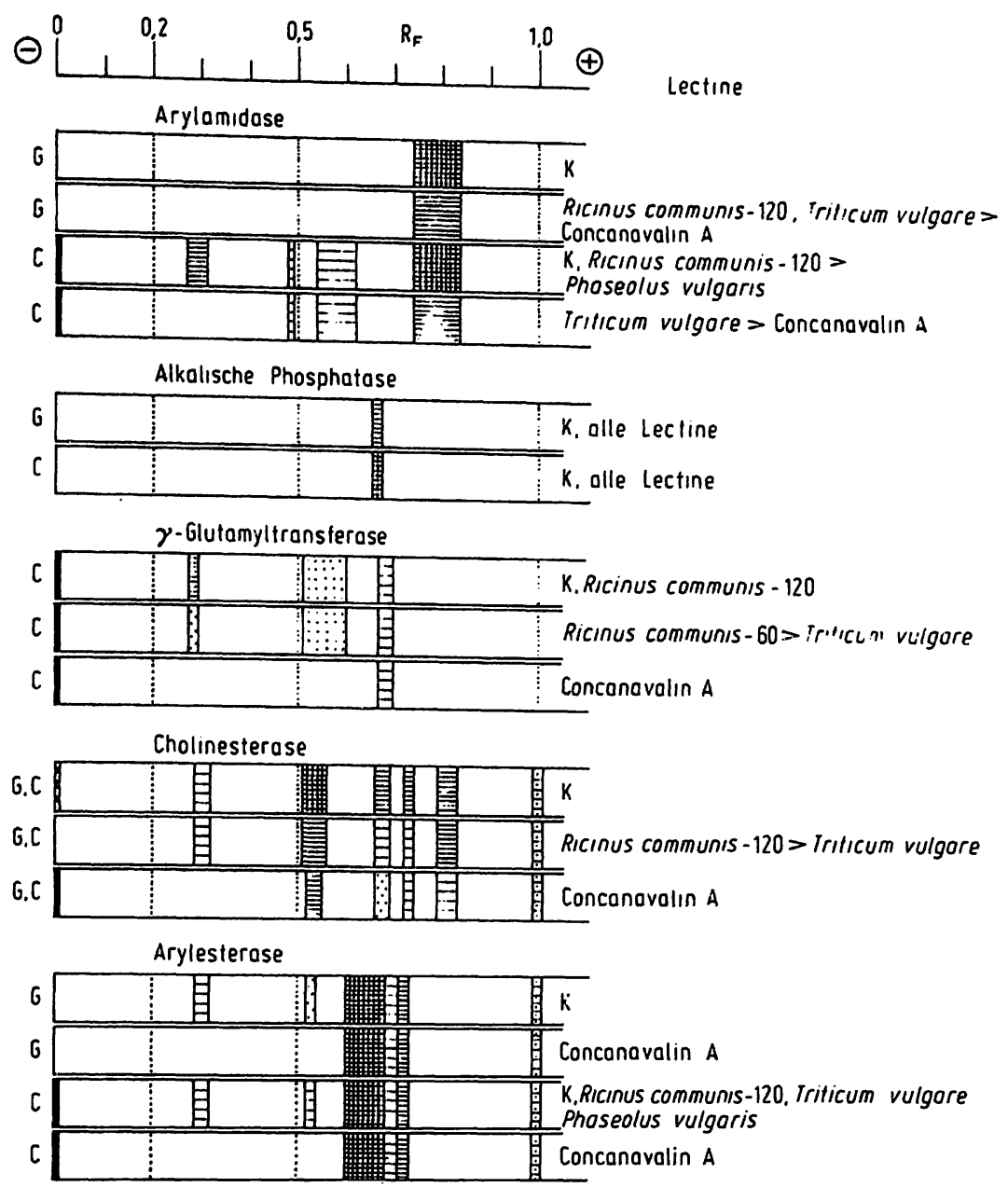

Abb. 3. Pherogramme multipler Formen von Enzymen aus Überständen von Poolseren Gesunder (G) und Kranker mit Lebermetastasen (C) nach Fällung mit Lectinen. Enzymaktivität im Polyacrylamidgel bezeichnet nach der Position des Albumins

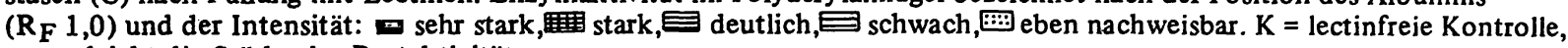
$>$ vergleicht die Stärke der Restaktivität.

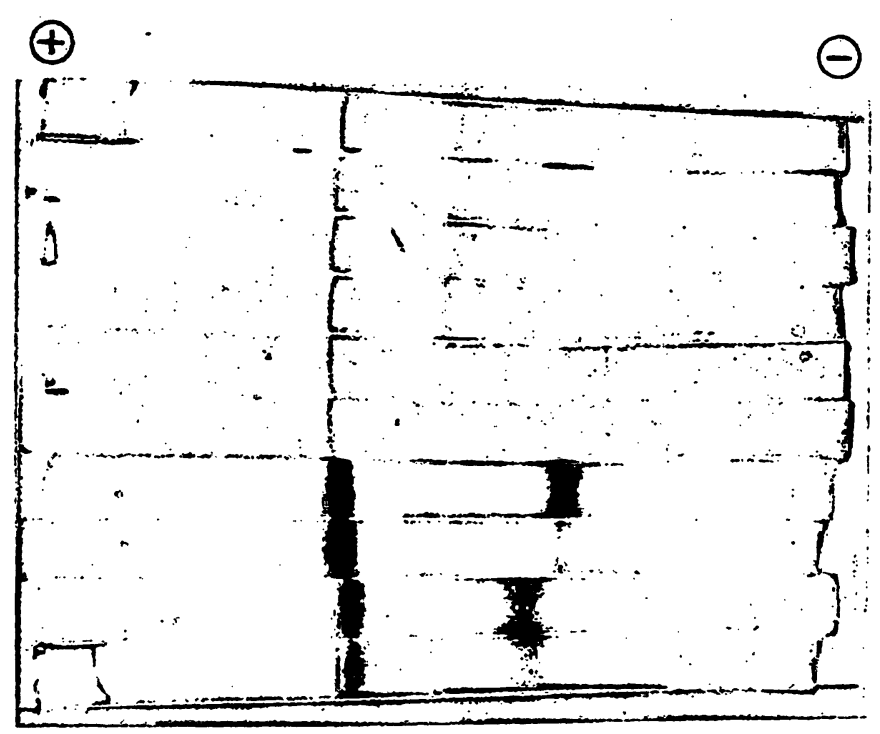

Abb. 4. Zymogramme aus den Überständen lectingefällter Seren von Ǩrebskranken mit Lebermetastasen. Von oben nach unten: Kontrolle, Canavalia ensiformis, Phaseolus vulgaris- und Triticum vulgare-Lectin (sämtlich Arylamidase);

Kontrolle, Concanavalin A (beide $\gamma$-Glutamyltransferase); Kontrolle, Concanavalin A (beide Cholinesterase) Kontrolle, Concanavalin A (beide Arylesterase). Kathode rechts, Vorgehen s. Methodik. oder nach Farbumschlag in Trichloressigsäure-Dimethylformamid. Im übrigen verhielten sich beide Esterasen verschieden: Die 7 Komponenten der Cholinesterase reagierten in den Seren von Gesunden und Krebskranken identisch. Ihre Zahl und Intensität wurden durch Concanavalin $\mathrm{A}$ und die Lectine aus Ricinus communis 120 und Triticum vulgare nur gering reduziert.

Im Serum Gesunder stellten sich 4-5 Arylesterase-Fraktionen dar, da sich bei hoher Aktivität die Banden 2 und 3 vereinigten. Bei Vorliegen von Lebermetastasen erschien, auch nach Reaktion mit Lectinen, zusätzlich Aktivität am Start (Abb. 3).

\section{Diskussion}

Die Deutung aller Ergebnisse muß folgende Versuchsbedingungen berücksichtigen:

1. Die mit den untersuchten Enzymen reagierende Lectinkonzentration ist unbekannt, da ein größerer Anteil von anderen Glykoproteinen des Serums verbraucht wird.

2. Vergleiche mit dem Verhalten gereinigter Enzyme sind problematisch. So wird Cholinesterase von Glycine max-Lectin ohne (6), im Serum aber erst nach Vorbehandlung mit Neuraminidase gebunden. Möglicherweise 
hemmt die noch hohe Proteinkonzentration im Reaktionsansatz die Fällung, so daß aktive Enzym-LectinKomplexe in Lösung bleiben. Hierauf deuten die am Gelanfang liegenden Fraktionen von Arylamidase, Cholinesterase und Arylesterase aus Überständen.

Dennoch sind Lectine zur Untersuchung der Heterogenität mikrosomaler Enzyme im Serum ebenso geeignet wie zur Charakterisierung hoch gereinigter Glykoproteine (29). Dies gilt vor allem für die in Seren von Carcinomträgern und Leberkranken deutlich schwächere Fällung von Cholinesterase durch Concanavalin A und von Arylamidase durch die Lectine aus Triticum vulgare und Phaseolus vulgaris. Gleichwohl betrifft die Präzipitationshemmung in diesen Seren nicht alle Lectine derselben Spezifität (Tab. 3).

Die Vorbehandlung mit Neuraminidase verändert in Seren von Gesunden und Krebskranken die Aktivität der Cholinesterase in entgegengegensetzter Weise, während sie die Aktivität der alkalischen Phosphatase, wie beim gereinigten Enzym aus menschlicher Leber (30), in beiden Poolseren erhöht und die der $\boldsymbol{\gamma}$-Glutamyltransferase mindert. Deren Verhalten ist durch das Vorliegen von 3 multiplen Formen im Serum, eines Lipid- und eines Proteinaggregats neben der freien $\boldsymbol{\gamma}$-Glutamyltransferase (31), besonders komplex.

Auch die Fällung der Enzyme zeigt nach der Abspaltung von N-Acetylneuraminsäure keine Gesetzmäßigkeit. Offenbar binden die Lectine, mit Ausnahme von Glycine max-Agglutinin, interne Zucker (32) ohne sterische Behinderung. So werden immer Mannose und N-Acetylglucosamin und gelegentlich $\mathrm{N}$-Acetylgalaktosamin erfaßt, während endständige Fucose-Reste sowie GalaktoseReste in vorletzter Position der Saccharidkette kaum reagieren. Nach Beobachtungen an alkalischer Phos- phatase aus dem Intestinum $(33,35)$ wird das fetale Sialoenzym sogar stärker von Concanavalin $\mathbf{A}$ gebunden als die adulte $\mathrm{N}$-acetylneuraminsäurefreie Form.

Diese Befunde lassen nicht entscheiden, ob bei onkogener Transformation durch extra- (35) und intrazelluläre Zunahme der Sialyltransferase (Übersicht bei 1.c. (2)) typischerweise nur Sialoenzyme (fetale $\gamma$-Glutamyltransferase: $2,4,7,20,36$ und fetale alkalische Phosphatase: 33) entstehen. Möglicherweise treten auch vermehrt Asialoenzyme (multiple Formen von Arylamidase: 15) durch Abnahme membrangebundener Glykosyltransferasen bei erhöhter intrazellulärer Glykosidaseaktivität (Übersicht bei 37) auf.

Dies lassen auch die Muster der Enzymfraktionen offen. Die in Seren von Malignomträgern erscheinenden kathodischen Banden entsprechen multiplen Formen mit niedrigem Gehalt an N-Acetylneuraminsäure. Die Lectine aus Canavalia ensiformis, Triticum vulgare, Phaseolus vulgaris und Ricinus communis-Agglutinin 120 reagieren mit ihnen bevorzugt, aber nicht ausschließlich. Dieses Verhalten gilt nicht für höhere Konzentrationen. Arylesterase ausgenommen, fällen sie alle Fraktionen, so daß dann die typischen Muster bei Carcinomen verschwinden. Nur die intensive Darstellung von Arylamidase, $\boldsymbol{\gamma}$-Glutamyltransferase, Cholinesterase und Arylesterase am Geleintritt deutet auf lösliche große Enzym-LectinKomplexe, die bei Gesunden fehlen.

$\mathrm{Ob}$ die Reaktion von gereinigter $\boldsymbol{\gamma}$-Glutamyltransferase aus menschlicher Leber und Niere (39) und von alkalischer Phosphatase aus Organextrakten (40) mit Lectinen eine Zuordnung ihrer fällbaren Anteile im Serum gestattet, sollen weitere Studien mit menschlichen Geweben entscheiden.

\section{Literatur}

1. Sharon, N. \& Lis, H. (1972), Science 177, 949-959.

2. Köttgen, E. (1977), Klin. Wochenschr. 55, 359-373.

3. Rüdiger, H. (1978), Naturwissenschaften 65, 239-244.

4. Köttgen, E. \& Gerok, W. (1976), Klin. Wochenschr. 54, 439-444.

5. Zygowicz, E. R., Sunderman, F. W., Horak, E. \& Dooley, J. F. (1977), Clin. Chem., 23, 2311-2323.

6. Uhlenbruck, G., Haupt, H., Reese, J. \& Steinhausen, G. (1977), diese Z. 15, 561-564.

7. Köttgen, E., Lindinger, G. \& Reutter, W. (1977), Clin. Chim. Acta $80,221-224$.

8. Goldstone, A., Konecny, Ph. \& Koenig, H. (1971), FEBS Lett. 13, 68-72.

9. Lorentz, K., Koch, C.-D., Flatter, B. \& Molz, J. (1975), diese Z. 13, 49-52.

10. Hausamen, T. U., Helger, R., Rick, W. \& Gross, W. (1967), Clin. Chim. Acta 15, 241-245.

11. Szasz, G., Weimann, G., Stähler, F., Wahlefeld, A. W. \& Persijn, J. P. (1974), diese Z. 12, 228.

12. Knedel, M. \& Böttger, R. (1967), Klin. Wochenschr. 45, $325-327$.

13. Lorentz, K., Flatter, B. \& Augustin, E. (1979), Clin. Chem. 25 (in press).
14. Lorentz, K., Flatter, B. (1977), diese Z. 15, 101-108.

15. Lorentz, K., Marunowski, A. \& Ritter, U. (1974), diese Z. $12,468-473$.

16. Rosenblum, D., Himmelhoch, S. R., Peterson, E. A., Evans, W. H. \& Mage, M. G. (1970), Arch. Biochem. Biophys. 141, 303-312.

17. Burlina, A. (1978), Clin. Chem. 24, 502-504.

18. Holmes, R. S. \& Masters, C. J. (1967), Biochim. Biophys. Acta 132, 379-399.

19. Kocourek, J., Jamieson, G. A., Votruba, T. \& Hořejצi, V. (1977), Biochim. Biophys. Acta 500, 344-360.

20. Köttgen, E., Prellwitż, W. \& Gerok, W. (1978), Verh. Dtsch. Ges. Inn. Med. 84, 1062-1064.

21. Miyazaki, S. \& Okumura, M. (1972), Clin. Chim. Acta 40, 193-197.

22. Patel, S. \& O'Gorman, P. (1973), Clin. Chim. Acta 49, $11-17$.

23. Kok, P. J. M. J., Seidel, B., Holtkamp, H. C., \& Huisman, J.'(1978), Clin. Chim. Acta 90, 209-216.

24. Dalal, F. R. \& Winsten, S. (1973), Clin. Chem. 19, 655.

25. Gaffney jr., P. J. (1970), Biochim. Biophys. Acta 207 , 465-476.

26. Holt. S. J. (1952), Nature 169; 271-273. 
27. Juul, P. (1968), Clin. Chim. Acta 19, 203-213.

28. Neuhoff, V. (1973), Micromethods in Molecular Biology. p. 14, Springer Verlag, Berlin-Heidelberg-New York.

29. Uhlenbruck, G., Baldo, B. A., Steinhausen, G., Schwick, H. G., Chatterjee, B. P., Hořejši, V., Krajhanzl, A. \& Kocourek, J. 1978), diese Z. 16, 19-23.

30. Komoda, T. \& Sakagishi, Y. (1976), Biochim. Biophys. Acta $438,138-152$.

31. Huseby, N. E. (1978), Biochim. Biophys. Acta 522, 354-362.

32. Goldstein, I. J., Reichert, C. M. \& Misaki, A. (1974), Ann. N. Y. Acad. Sci. 234, 283-296.

33. Higashino, K., Otani, R., Kudo, S. \& Yamamura, Y. (1977), Clin. Chem. 23, 1615-1623.
34. Komoda, T. \& Sakagishi, Y. (1978), Biochim. Biophys. Acta $523,395-406$.

35. Ganzinger, U., Dorner, F., Unger, F. M., Moser, K. \& Jentzsch, K. (1977), Klin. Wochenschr. 55, 553-555.

36. Köttgen, E. \& Lindinger, G. (1976), Hoppe-Seyler's Z. Physiol. Chem. 357, 1439-1442.

37. Hanfland, P. \& Uhlenbruck, G. (1978), diese Z. 16 85-105.

38. Böhme, J., Hütter, H. J., Gerlach, W. \& Haschen, R. J. (1976), Enzyme 21, 464-470.

39. Shaw, L. M., London, J. W. \& Petersen, L. E. (1978), Clin. Chem. 24, 905-915.

40. Chang, C.-H., Angellis, D. \& Fishman, W. H. (1975), Mol. Cell. Biochem. 9, 55-57.
Prof. Dr. Klaus Lorentz Kronsforder Allee 71-73 2400 Lübeck 

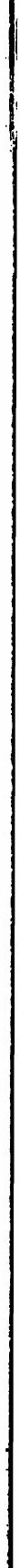